\title{
ГРУППОВОЙ ПОДХОД В ДИНАМИКЕ МНОГОСПИНОВЫХ СИСТЕМ. V
}

Система, состоящая из одного спина $1 / 2$, - простейшая двухуровневая квантовая система. В данной статье на ее примере выясняются детали группового подхода в квантовой динамике. Представления исследуемой динамической группы $S U(2)$ имеют множество приложений и в динамике многоспиновых систем.

До сих пор динамика системы изучалась применительно к некоторым встречающимся в практике видам временной зависимости внешнего магнитного поля. В данной статье рассматривается общий случай произвольно изменяющегося поля. Устанавливается связь между временным изменением состояннй системы (либо ансамбля систем), с одной стороны, и управляющим этим движением магнитным полем (или гамильтонианом) - с другой. Специально обсуждаются пути обобщения понятия резонанса.

Статья автономна. Для ее понимания требуется, помимо общих положений теории непрерывных групп $\left[{ }^{1-3}\right]$, знакомство с вводной статьей этой серии [4].

\section{9. Динамика двухуровневой системы}

9.1. Группа $\mathbf{u}_{2}$ и е с спероператорное представлени е. Пространство С (чистых) квантовых состояний, по определению, 2 -мерно. Если $a_{m}$-базис пространства C $\left[{ }^{4}\right]$ составлен из координатных строк $a_{1}=[1,0]$ и $a_{2}=[0,1]$, то любой (нормированный) вектор состояния $x \in \mathbf{C}$ может быть представлен в виде суперпозиции

$$
x\left(\Phi, \varphi_{1}, \varphi_{2}\right)=\cos \Phi \mathrm{e}^{-i \varphi_{1}} a_{1}+\sin \Phi \mathrm{e}^{-i \varphi_{2}} a_{2} .
$$

Угловые параметры $\left(\Phi, \varphi_{1}, \varphi_{2}\right)$ векторов состояний имеют следующие пределы изменения

$$
0 \leqslant \Phi \leqslant \pi / 2 ; \quad-\pi \leqslant \varphi_{1}, \varphi_{2} \leqslant \pi .
$$

Однако формулы (9.2) требуют некоторых разъяснений, чтобы понять особенность топологии множества векторов (9.1).

Рассмотрим подмножество векторов (9.1) с постоянным Ф и переменными $\varphi_{1}$ и $\varphi_{2}$. Существуют три различных типа таких подмножеств:

a) $0<\Phi<\pi / 2-$ поверхность тора,

б) $\Phi=0-$ круг (только переменная $\left.\varphi_{1}\right)$,

в) $\Phi=\pi / 2-$ круг (только переменная $\varphi_{2}$ ).

Итак, в случаях б) и в) лишь один из параметров $\varphi_{1}, \varphi_{2}$ имеет смысл, второй считаем нулем. 
Если система содержит независящий от времени гамильтониан $H_{0}$ с собственными векторами $a_{m}(m=1,2)$, то состояния б) и в) называются стационарными, а состояния а) - нестационарными. Однако с математической точки зрения базис $a_{m}$ не является особым. Аналогичное «расслоение» на множества квантовых состояний дает любой базис пространства С.

Углы (9.2) могут быть использованы для параметризации действующих в $\mathbf{u}_{2}{ }^{*}$ унитарных унимодулярных операторов $T\left(\Phi, \varphi_{1}, \varphi_{2}\right)$. Матрица любого такого оператора (на $a_{m}$-базисе) имеет вид

$$
T\left(\Phi, \varphi_{1}, \varphi_{2}\right)=\left[\begin{array}{ll}
\cos \Phi \mathrm{e}^{-i \varphi_{1}}-\sin \Phi \mathrm{e}^{i \varphi_{2}} \\
\sin \Phi \mathrm{e}^{-i \varphi_{2}} & \cos \Phi \mathrm{e}^{i \varphi_{1}}
\end{array}\right] .
$$

Между векторами (9.1) и операторами (9.3) существует взаимно однозначное соответствие

$$
x\left(\Phi, \varphi_{1}, \varphi_{2}\right)=T\left(\Phi, \varphi_{1}, \varphi_{2}\right) a_{1},
$$

указывающее на совпадение топологии группы $\mathbf{u}_{2}$ и множества векторов (9.1). Значит, квантовые состояния двухуровневой системы можно выразить с помощью операторов состояний $(9.3)$, т. е. всю динамику $\mathbf{u}_{2}$ описывать на групповом языке. Более того, за представителя квантовых состояний можно принимать риманово пространство точек $\left(\Phi, \varphi_{1}, \varphi_{2}\right)$.

Различие этих трех способов описания квантовых состояний состоит в применяемой алгебре. В случае векторов (9.1) - это линейная алгебра над комплексным пространством С. В случае операторов (9.3) - матричная алгебра. Углы же $\left(\Phi, \varphi_{1}, \varphi_{2}\right)$ подчиняются более сложной алгебре. Для описания перемещения системы применима только групповая операция (умножение) матриц (9.3). Линейные операции этих матриц хотя и полезны при установленни связи с динамическим кольцом $\left[{ }^{4}\right]$, но не обязательны $\left[{ }^{1}\right]$. Можно себе представить более общие динамики, описываемые группами Ли. Но если эти группы компактны (пространственно локализованные системы?), то они неминуемо пмеот унитарное представление $\left[{ }^{1}\right]$.

Пространство всех линейных операторов О 4-мерно. Поставим в соответствие $a_{m}$-базису пространства $\mathbf{C} A$-базис пространства $\mathbf{O}\left[{ }^{4}\right]$. Образуем эрмитовый $A$-базис $A_{11}, A_{22}, X_{12}, Y_{12}$, что и даст основу 4-мерного пространства $\mathbf{H}$ эрмитовых операторов. В целях выделения 3-мерного подпространства $\mathbf{H}^{0} \subset \mathbf{H}$ операторов с нулевым следом видоизменим эрмитовый $A$-базис следующим образом:

$$
\begin{gathered}
I_{0}=1 / 2\left(A_{11}+A_{22}\right) ; \\
I_{z}=Z_{12}=1 / 2\left(A_{11}-A_{22}\right), \\
I_{x}=X_{12}=1 / 2\left(I_{+}+I_{-}\right), \\
I_{y}=Y_{12}=-i / 2\left(I_{+}-I_{-}\right),
\end{gathered}
$$

где $I_{+}=A_{12}$ и $I_{-}=A_{21}$. Стало быть,

$$
I_{2} a_{m}=\mu_{m} a_{m}
$$

где $\mu_{1}=+1 / 2$ и $\mu_{2}=-1 / 2$. В данном случае $I$-базис $\left[{ }^{4}\right]$ динамического кольца $\mathbf{u}_{2}{ }^{0}$ совпадает с (видоизмененным) эрмитовым $A$-базисом.

* Вместо более распространенного в физике обозначения $S U(2)$ [1] мы занмствуем обозначения из $\left[{ }^{2}\right]$. 
Базисные операторы (9.6) подчиняются алгебре Ли $\mathbf{u}_{2}{ }^{0}$. Метрика в 3 -мерном евклидовом пространстве $\mathbf{H}^{0}$ определяется скалярными произведениями

$$
\left(I_{j}, I_{k}\right)=1 / 2 \delta_{j k} \quad(j, k=z, x, y) .
$$

Любой оператор вида

$$
I_{n}=\sum_{j} n_{j} I_{j} \in \mathbf{H}^{0}, \quad \sum_{j} n_{j}^{2}=1
$$

называется спиновым оператором. Все они имеют одинаковые собственные значения $\mu_{m}$, но различные собственные векторы. Любой эрмитовый оператор $H \subset \mathbf{H}^{0}$ может быть представлен в виде

$$
H=\omega_{12} I_{n}=\sum_{j} \omega_{j} I_{j},
$$

где $\omega_{j}=n_{j} \omega_{12}$. Оператор $(9.10)$ имеет собственные значения $\omega_{m}=$ $=\mu_{m} \omega_{12}$. Символ $\omega_{12}=\omega_{1}-\omega_{2}$ обозначает (угловую) частоту единственного перехода $1 \rightarrow 2$.

Ортонормированный репер $\vec{a}_{j}(j=z, x, y)$, связанный с измерительной аппаратурой (лабораторный репер), выделяет 3-мерное (действительное) векторное пространство $\mathbf{V}$. Векторная алгебра V изоморфна алгебре $\mathbf{u}_{2}{ }^{0}$. Взаимно однозначное соответствие линейных операций в $\mathbf{V}$ и $\mathbf{u}_{2}{ }^{0}$ вытекает из сопоставления единичного вектора направления

$$
\vec{n}=\sum_{j} n_{j} \vec{a}_{j} \in \mathrm{V}
$$

и спинового оператора (9.9). Векторному произведению $\left[\vec{H}_{1}, \vec{H}_{2}\right]$ соответствует коммутатор $-i\left[H_{1}, H_{2}\right]$, а скалярные произведения связаны соотношением

$$
\left(\vec{H}_{1}, \vec{H}_{2}\right)=2\left(H_{1}, H_{2}\right) .
$$

Появляется возможность говорить о пространственном направлении операторов (9.9) и (9.10). Какой физический смысл вкладывается в операторы (9.9), зависит от природы двухуровневой системы. Она может быть, например, изоспином или же т. н. энергетическим спином [ $\left.{ }^{5}\right]$.

Принимая двухуровневую систему за ядерный спин $1 / 2$, приписываем ей (как источнику магнитного поля) оператор ядерного магнитного момента

$$
M_{m}=\gamma \hbar I_{m}
$$

в направлении $\vec{m}$. Вещество, содержащее в единице объема $N$ спинов и трактуемое как больцмановский ансамбль спинов, будет обладать ядерной намагниченностью

$$
\vec{M}=N\left(\varrho, M_{m}\right) \vec{m} \in \mathbf{V}
$$

если состояние ансамбля задано оператором плотности $Q \in \mathbf{H}$.

С другой стороны, ядерный спин взаимодействует с внешним магнитным полем $\vec{B}=\overrightarrow{B n} \in \mathbf{V}$ согласно гамильтониану зеемановского взаимодействия (9.10). При этом

$$
\omega_{12}=-\gamma B
$$


- ларморовая частота, а $\gamma$ - гиромагнитное отношение ядерного спина.

Состояния ансамбля спинов $1 / 2$ описываются оператором плотности

$$
\varrho=I_{0}+\pi_{12} I_{m} \in \mathbf{H} .
$$

Эрмитовый оператор (9.16) имеет собственные векторы $s_{m} \in \mathbf{C}$ и собственные значения (естественные популяции) $0 \leqslant \pi_{m} \leqslant 1$ :

$$
\mathrm{Q} s_{m}=\pi_{m} s_{m} .
$$

Разница $\pi_{12}=\pi_{1}-\pi_{2}$ именуется поляризацией.

$\mathrm{B}$ необратимых процессах типа спиновой релаксации, химической и динамической поляризации $\pi_{12}$ может изменяться во времени. В рассматриваемых нами обратимых процессах поляризация сохраняется. Поэтому состояния ансамбля достаточно описывать спиновыми операторами $I_{m} \in \mathbf{H}^{0}$, образующими сферу направлений $\vec{m}$. Тогда ядерная намагниченность вычисляется по формуле

$$
\vec{M}=(1 / 2) \gamma \hbar N \pi_{12} \vec{m}
$$

Унитарное супероператорное представление $\left[{ }^{4}\right]$ операторов (9.3) в. пространстве $\mathbf{0}$ получается путем составления 4 X 4 матриц супероператоров на $A$-базисе. Преобразование этих матриц на $I$-базис приводит к ортогональному супероператорному представлению $\left[{ }^{4}\right]$ группы $\mathbf{u}_{2}$ в пространстве $\mathbf{H}$

$$
T\left(\Phi, \varphi_{1}, \varphi_{2}\right) \rightarrow \mathfrak{I}\left(\Phi, \varphi_{1}, \varphi_{2}\right)
$$

в виде $4 \times 4$ ортогональных матриц с $\operatorname{det} \mathfrak{I}=1$. Как одномерное подпространство, натянутое на $I_{0}$, так и 3 -мерное подпространство $\mathbf{H}^{0}$ являются инвариантными относительно представления (9.19).

9.2. Әйл еров а п а а метризация. В целях разложения операторов (9.3) на составляющие, зависящие только от одного параметра, заменим углы (9.2) (первичные параметры) новыми (эйлеровыми) параметрами $\left(\varphi_{12}, \Phi, \sigma_{12}\right)$. В новой параметризации $Ф$ сохраняет прежний смысл, но

$$
\begin{aligned}
& \varphi_{12}=\varphi_{1}-\varphi_{2}, \\
& \sigma_{12}=\varphi_{1}+\varphi_{2} .
\end{aligned}
$$

В дальнейшем углы (9.20) и (9.21) будут иметь различный смысл в зависимости от того, являются ли они параметрами группы $\mathbf{u}_{2}$ или ее супероператорного представления. В первом случае они могут изменяться в пределах

$$
-2 \pi \leqslant \varphi_{12}, \quad \sigma_{12} \leqslant 2 \pi .
$$

Допускаются лишь такие пары, которые имеют образ $\varphi_{1}, \varphi_{2}$.

Во втором случае пределы изменения углов $\varphi_{12}$ и $\sigma_{12}$ обычные, т. е. $0 \leqslant \varphi_{12}, \quad \sigma_{12} \leqslant 2 \pi$.

$\mathrm{B}\left[{ }^{3}\right]$ применена несколько иная параметризация унитарных групп. Наш выбор обусловлен желанием сохранить непрерывность параметров при их независимом изменении.

Любой оператор $T\left(\varphi_{12}, \Psi, \sigma_{12}\right) \in \mathbf{u}_{2}$ типа (9.3) может быть представлен в виде произведения трех экспонент

$$
T\left(\varphi_{12}, \Phi, \sigma_{12}\right)=T_{1}\left(\varphi_{12}\right) T_{2}(\Phi) T_{3}\left(\sigma_{12}\right),
$$


где

$$
\begin{aligned}
& T_{1}\left(\varphi_{12}\right)=\exp \left(-i \varphi_{12} I_{z}\right), \\
& T_{2}(\Phi)=\exp \left(-i 2 \Phi I_{y}\right), \\
& T_{3}\left(\sigma_{12}\right)=\exp \left(-i \sigma_{12} I_{z}\right) .
\end{aligned}
$$

Применение оператора (9.23) в формуле (9.4) приводит к эйлеровой параметризации векторов состояния $x\left(\varphi_{12}, \Phi, \sigma_{12}\right)$.

Аналогично (9.23) разлагаются супероператоры $\mathfrak{I}\left(\varphi_{12}, \Phi, \sigma_{12}\right)$ представления (9.19):

где

$$
\mathfrak{I}\left(\varphi_{12}, \Phi, \sigma_{12}\right)=\mathfrak{I}_{1}\left(\varphi_{12}\right) \mathfrak{I}_{2}(\Phi) \mathfrak{I}_{3}\left(\sigma_{12}\right),
$$

$$
\begin{aligned}
\mathfrak{I}_{1}\left(\varphi_{12}\right) & =\Re\left(I_{2}, \varphi_{12}\right), \\
\mathfrak{I}_{2}(\Phi) & =\Re\left(I_{y}, 2 \Phi\right), \\
\mathfrak{I}_{3}\left(\sigma_{12}\right) & =\Re\left(I_{z}, \sigma_{12}\right) .
\end{aligned}
$$

В формулах $(9.28)-(9.30)$ содержится укороченная символика ортогональных супероператоров, которую отныне будем использовать. Например, супероператор (9.29) совершает правое вращение вокруг оси $I_{y}$ на угол $2 \Phi$, оставляя $I_{0}$ неизменным.

Эйлерова параметризация спиновых операторов $I_{m}\left(\varphi_{12}, \Phi\right)$ состояний ансамбля (9.16) получается по формуле

$$
I_{m}\left(\varphi_{12}, \Phi\right)=\mathfrak{I}\left(\varphi_{12}, \Phi, \sigma_{12}\right) I_{z}=\mathfrak{I}_{1}\left(\varphi_{12}\right) \mathfrak{I}_{2}(\Phi) I_{z} .
$$

Состояния ансамбля зависят только от эйлеровых параметров $\varphi_{12}$ и $2 Ф-$ двух сферических координат направления $\vec{m}\left(\varphi_{12}, 2 \Phi\right) \in \mathbf{V}$.

Становится явной неизоморфность представления (9.19). Левое вращение, описываемое отрицательными углами $\varphi_{12}$, всегда эквивалентно некоторому правому вращению. Более того, $\sigma_{12}$ выпадает из (9.31).

Группа операторов (9.27) представляет собой приводимое представление группы вращений $\mathbf{d}_{3}$ (т. е. $\left.S O(3)\right)$. Преобразование

$$
\mathfrak{I}\left(\varphi_{12}, \Phi, \sigma_{12}\right) I_{j} \quad(j=z, x, y),
$$

совершаемое операторами (9.27), является эйлеровым вращением лабораторного репера (9.6). Углы $\varphi_{12}, 2 \Phi$ и $\sigma_{12}-$ это углы прецессии, нутации и собственного вращения соответственно. Обратное к (9.19) соответствие будет многозначным представлением группы $\mathbf{d}_{3}$.

Согласно $(9.16)$ и $(9.17)$ операторы $\varrho\left(\varphi_{12}, \Phi\right)$ и $I_{m}\left(\varphi_{12}, \Phi\right)$ имеют общую систему собственных векторов $s_{m}\left(\varphi_{12}, \Phi, \sigma_{12}\right)(m=1,2)$. В силу (9.31) и (9.19) верно

$$
s_{m}\left(\varphi_{12}, \Phi, \sigma_{12}\right)=T\left(\varphi_{12}, \Phi, \sigma_{12}\right) a_{m} .
$$

Согласно (9.4), вектор $s_{1}\left(\varphi_{12}, \Phi, \sigma_{12}\right)$ - представитель произвольного $x\left(\varphi_{12}, \Phi, \sigma_{12}\right) \in \mathbf{C}$.

Каждому состоянию ансамбля $I_{m}\left(\varphi_{12}, \Phi\right)$ соответствует подмножество состояний $s_{1}\left(\varphi_{12}, \Phi, \sigma_{12}\right)$, обладающее следующим свойством: намагниченности, вычисленные по формуле квантового среднего

$$
\vec{M}=N\left\langle M_{m}\right\rangle \vec{m}
$$

и по формуле (9.14), совпадают, если только $\pi_{12}=1$ (все спины в состояниях $s_{1}\left(\varphi_{12}, \Phi, \sigma_{12}\right), \sigma_{12}$ - переменная). 
9.3. Динамика $\mathbf{u}_{2}$. Квантовая динамика ядерного спина построена по следующей общей схеме

$$
\vec{B}(t) \rightarrow \overline{\mid H(t) \rightarrow x(t) \rightarrow \varrho(t)} \rightarrow \vec{M}(t) .
$$

Классическое (внешнее) магнитное поле $\vec{B}(t)$ выступает здесь в роли входного (управляющего) сигнала, намагниченность $\vec{M}(t)-$ в роли выходного (классического наблюдаемого). В раме - «черный ящик квантового формализма».

Переход от $x(t)$ к $\varrho(t)$ предусматривает введение статистики ансамбля. Вышеотмеченная неизоморфность представления (9.19) является (дополнительной) «квантовой частью» возникающей убыли информации о состоянии системы. Теряются «квантовые корреляции» спина и магнитного поля (классическое поле взамен квантованного).

Исследователь, интересующийся только измерением $\vec{M}(t)$, может обойти стадию вычисления $x(t)$ (прямой метод в теории магнитного резонанса).

Под прямой задачей динамики (задачей управления спином) подразумеваем следующее: дано $x(t)$ (либо только $I_{m}(t)$ ), требуется найти возможные $\vec{B}(t)$, управляющие этим движением. Решением этой задачи является сформулированное ниже понятие подсемейства траекторий.

Обратная задача динамики гласит: даны $x(0)$ (либо только $I_{m}(0)$ ) и $\vec{B}(t)$, требуется найти $x(t)$ и сигнал $\vec{M}(t)$. Компактным решением этой задачи служит семейство траекторий, управляемое одним и тем же $\vec{B}(t)\left[{ }^{4}\right]$.

При групповом подходе $\left[{ }^{4}\right]$ «черный ящик» из (9.35) заменяется системой соотношений

$$
H(t) \leftrightarrow D(t, 0) \rightarrow \Re(t, 0) .
$$

Преобразование движения $D(t, 0) \in \mathbf{u}_{2}$ описывает семейство траекторий

$$
x(t)=D(t, 0) x(0) \in \mathbf{C},
$$

управляемое гамильтонианом $H(t)$, а ортогональное супероператорное представление $\Re(t, 0)$ оператора $D(t, 0)$ - соответствующее суженное семейство

$$
I_{m}(t)=\Re(t, 0) I_{m}(0) \in \mathbf{H}^{0} .
$$

Установление входящих в (9.36) матриц - один из возможных способов описания семейства $\left[{ }^{4}\right]$. При этом о векторах $x, I_{m}$ поставляют данные их компоненты на базисе $a_{m}$ или $I_{j}$ соответственно. Второй способ описания семейства - составление списка входящих в семейство подсемейств. При этом способе траектории даются своими эйлеровыми параметрами. Ниже рассматриваются оба способа в их взаимосвязи.

Введение временной зависимости в эйлеровы параметры

$$
\left(\varphi_{12}(t), \Phi(t), \sigma_{12}(t)\right)
$$

определяет траектории системы

$$
x(t)=T(t) a_{1}
$$

и соответствующие им траектории ансамбля

$$
I_{m}(t)=\widetilde{I}(t) I_{z} .
$$


Подстановка (9.39) в формулы $(9.23)-(9.26)$ и $(9.27)-(9.30)$ позволяет записать явные выражения $T(t)$ и $\mathfrak{\mathcal { L }}(t)$ в виде разложений

$$
\begin{aligned}
& T(t)=T_{1}(t) T_{2}(t) T_{3}(t), \\
& \mathfrak{I}(t)=\mathfrak{I}_{1}(t) \mathfrak{I}_{2}(t) \mathfrak{I}_{3}(t) .
\end{aligned}
$$

Траектории (9.39) различаются начальными состояниями $\left(\varphi_{12}(0), \Phi(0), \sigma_{12}(0)\right)$ и дифференциальными частотными характеристиками

$$
\begin{aligned}
& v_{1}(t)=d \varphi_{12} / d t=d \alpha_{1} / d t, \\
& v_{2}(t)=2 d \Phi / d t=2 d \alpha_{2} / d t, \\
& v_{3}(t)=d \sigma_{12} / d t=d \alpha_{3} / d t,
\end{aligned}
$$

(учитываем пока только первые части этих равенств).

Эквивалентные траекториям (9.40) траектории (9.42) тоже подчиняются уравнению Шредингера

$$
i \frac{d T}{d t}=H(t) T
$$

с тем же гамильтонианом $H(t)$, что и $x(t)$. Дифференцированием получим

$$
H(t)=v_{1}(t) I_{z}+v_{2}(t) I_{y}(t)+v_{3}(t) I_{m}(t),
$$

где

$$
\begin{aligned}
I_{j}(t) & =\mathfrak{I}_{1}(t) I_{j} \quad(j=x, y), \\
I_{m}(t) & =\mathfrak{I}_{1}(t) \mathfrak{I}_{2}(t) I_{z} .
\end{aligned}
$$

Объединим в одно подсемейство те траектории $x(t)$, у которых одинаковые частоты $(9.44)-(9.46)$ и начальные состояния которых соответствуют одной и той же $I_{m}(0)$. Все траектории подсемейства (и только они) обеспечивают один и тот же сигнал $\vec{M}(t)$ в смысле $(9.34)$. Траектории подсемейства управляются одним и тем же гамильтонианом (9.48) .

В силу отмеченного в п. 9.2 двоякого смысла углов (9.20) подсемейство распадается на две непрерывные половины. Начальные параметры $\varphi_{12}(0)$ этих половин отличаются на $2 \pi$. Значения $\varphi_{12}(0), \Phi(0)$ являются общими для одной половины подсемейства. Параметр $\sigma_{12}(0)-$ переменная подсемейства.

Вместе с $I_{m}(t)$ к подсемейству примыкают их собственные векторы (9.33). Угол $\sigma_{12}(t)-$ разница их фаз. Подсемейство состоит из векторов $x(t)=s_{1}(t)$.

Траектории подсемейства управляются определенным гамильтонианом (9.48). Однако соответствующая подсемейству траектория $I_{m}(t)$ управляется множеством гамильтонианов (9.48), могущйх отличаться частотой $v_{3}(t)$. Относительно вращающегося репера (9.49) гамильтониан $(9.48)$ имеет вид

$$
H(t)=\omega_{z}(t) I_{z}+\omega_{x}(t) I_{x}(t)+\omega_{y}(t) I_{y}(t),
$$

где

$$
\begin{aligned}
& \omega_{z}(t)=v_{1}(t)+v_{3}(t) \cos 2 \Phi(t), \\
& \omega_{x}(t)=v_{3}(t) \sin 2 \Phi(t), \\
& \omega_{y}(t)=v_{2}(t) .
\end{aligned}
$$


Относительно лабораторного репера гамильтониан (9.51) может быть представлен в виде суммы продольной (относительно $I_{z}$ ) и вращающейся поперечной составляющих

$$
H(t)=H_{z}(t)+H_{\perp}(t),
$$

где

$$
\begin{gathered}
H_{z}(t)=\omega_{z}(t) I_{z}, \\
H_{\perp}(t)=\omega_{\perp}(t) \Re\left(I_{z}, \psi(t)\right) I_{x} .
\end{gathered}
$$

Тогда

$$
\begin{aligned}
& \omega_{x}(t)=\omega_{\perp}(t) \cos \left(\psi(t)-\varphi_{12}(t)\right), \\
& \omega_{y}(t)=\omega_{\perp}(t) \sin \left(\psi(t)-\varphi_{12}(t)\right) .
\end{aligned}
$$

В типичном эксперименте (по магнитному резонансу) $H_{z}(t)$ обязан своим происхождением сильному магнитному полю, а $H_{\perp}(t)-$ слабому полю возбуждения, т. е.

$$
\left|\omega_{\perp}(t)\right| \ll\left|\omega_{z}(t)\right| .
$$

В силу (9.44)-(9.46) уравнения (9.52) - (9.54) - дифференциальные. Их решения, при всех возможных начальных состояниях, и определяют список подсемейств, образующих семейство с гамильтонианом $(9.55)-(9.57)$.

Практически проблема сводится к решению нелинейных интегральных дифференциальных уравнений

$$
\begin{gathered}
d \varphi_{12} / d t=\omega_{z}(t)-\omega_{x}(t) \cot 2 \Phi(t), \\
\Phi(t)=\Phi(0)+\int_{0}^{t} \omega_{y}\left(t^{\prime}\right) d t^{\prime},
\end{gathered}
$$

где $\omega_{x}(t), \omega_{y}(t)$ берутся из $(9.58),(9.59)$. Заданными считаются: $\omega_{z}(t)$, $\omega_{\perp}(t)$ и $\psi(t)$.

Вышеописанный способ разложения унитарных операторов (и ортогональных супероператоров) на три составляющих, каждая из которых зависит только от одного параметра, приме́ним к входящим в (9.37), (9.38) преобразованиям движения семейства.

Назовем траекторию с начальным состоянием $\varphi_{12}(0)=\Phi(0)=$ $=\sigma_{12}(0)$ центральной. Эйлеровы параметры (9.39) этой траектории обозначим через

$$
\left(\alpha_{1}(\dot{t}, 0), \alpha_{2}(t, 0), \alpha_{3}(t, 0)\right) .
$$

Стало быть, $\alpha_{k}(0,0)=0(k=1,2,3)$.

Примем (9.63) за параметры преобразования движения семейства $\left[{ }^{4}\right]$. Заменим ими эйлеровы параметры в формулах $(9.23)-(9.26)$. Вместо $(9.42)$ запишем

$$
D(t, 0)=D_{1}(t, 0) D_{2}(t, 0) D_{3}(t, 0),
$$

а вместо $(9.48)-$

$$
H(t)=v_{1}(t) I_{2}+v_{2}(t) I_{y}^{\prime}(t)+v_{3}(t) I_{z}^{\prime \prime}(t) .
$$

Аналогично, взамен (9.43) имеем супероператорное представление оператора (9.64)

$$
\Re(t, 0)=\Re_{1}(t, 0) \Re_{2}(t, 0) \Re_{3}(t, 0),
$$


где

$$
\begin{aligned}
& \Re_{1}(t, 0)=\Re\left(I_{z}, \alpha_{1}(t, 0)\right), \\
& \Re_{2}(t, 0)=\Re\left(I_{y}, 2 \alpha_{2}(t, 0)\right), \\
& \Re_{3}(t, 0)=\Re\left(I_{z}, \alpha_{3}(t, 0)\right) .
\end{aligned}
$$

Тогда входящие в (9.65) спиновые операторы $I_{y}{ }^{\prime}(t), I_{z}^{\prime \prime}(t)$ определяются формулами

$$
\begin{gathered}
I^{\prime}{ }_{y}(t)=\Re_{1}(t, 0) I_{y}, \\
I_{z}^{\prime \prime}(t)=\Re_{1}(t, 0) \Re_{2}(t, 0) I_{z} .
\end{gathered}
$$

В случае центрального подсемейства $D(t, 0)$ - не только оператор состояния, но и преобразование движения. Вместе с тем (9.64) и. (9.66) - преобразования движения целого семейства, обладающего гамильтонианом $(9.65)$.

Мультипликативные разложения (9.64), (9.66) и аддитивное разложение (9.65) приспособлены к центральному подмножеству. Можно выписать аналогичные разложения, пригодные к любым другим подсемействам данного семейства. В таком случае гамильтониан будет иметь пписпособленный к подсемейству вид (9.48). нп с иными частотами (9.44)-(9.46), чем те. которые входят в (9.65). Каждый параметр (9.39) траектории выбранного подсемейства аддитивно слагается из значения начального состояния и значения соответствуюшего параметра ппеобразования движения (9.63) данного подсемейства. Последние соответствуют вторым частям равенств $(9.44)-(9.46)$.

Как геометрическая форма разных тпаекторий данного семейства, так и способ их временной параметризашии. воппше говоря. различны.

Множество всех пентральных траекторий $I_{z}^{\prime \prime}(t)$ всевозможных семейств содержит все простпанственно-временные формы динамики. Остальные тпаектории получаются из них путем смещения начального состояния $I_{m}(0)$.

Типичная центральная траектория $\left(\alpha_{1}(t, 0), 2 \alpha_{2}(t, 0)\right)$ - это некотопая винтовая линия на поверхности сферы $\alpha_{2}=f\left(\alpha_{1}\right)$. Уклон этой линии относительно «широтной»

$$
2 d \alpha_{2} / d \alpha_{1}=v_{2}(t) / v_{1}(t) .
$$

В vсловиях (9.60) этот уклон очень мал, и линня плотно наматывает cळenv (полностью или частично), образуя замкнутую или незамкнутую кривую.

Замкнутая кривая получается тогда. когда моменты времени $0, t_{1}$. t..... разделяют временную шкалу на такие интервалы, что отношения типа

$$
\int_{t_{1}}^{t_{2}} v_{2}\left(t^{\prime}\right) d t^{\prime} / \int_{t_{1}}^{t_{2}} v_{1}\left(t^{\prime}\right) d t^{\prime}
$$

будут рациональными числами. В противном случае имеем бесконечную «иррациональную обмотку сферы».

Выше предполагалось, что $v_{1}, v_{2}$ сохраняют знак и интегралы в (9.73) кратны 2 . В этом случае замкнутая винтовая линия начинается с $I_{z}$, доходит до $-I_{z}$ и возвращается к концу интервала к $I_{z}$.

В случае знакопеременного $v_{2}(t)$ возможны более сложные (но легко представляемые) формы траекторий. 
9.4. Р езонанс. Если взаимодействие (9.56) сильно в смысле (9.60), то действие возбуждения (9.57) на движение заметно лишь в том случае, когда частота возбуждения близка к частоте резонанса

$$
v(t)=\omega_{z}(t),
$$

где

$$
v(t)=d \psi / d t
$$

Расстройка

$$
\Delta v(t)=\omega_{z}(t)-v(t)
$$

частоты возбуждения (9.75) поряддка $\left|\omega_{\perp}(t)\right|$ (область резонанса).

Поясним сказанное путем последовательного рассмотрения некоторых специальных случаев.

Свободная прецессия. Возбуждение (9.57) отсутствует. По (9.52)(9.54) имеем

$$
v_{1}(t)=\omega_{0}(t) ; \quad v_{2}(t)=v_{3}(t)=0 .
$$

Сигнал

$$
M_{x}(t)=M_{x}(0) \cos \int_{0}^{t} \omega_{z}\left(t^{\prime}\right) d t^{\prime}
$$

может быть частотно модулированным. Движение происходит по $\varphi_{12-}$ линиям.

Обобщенный резонанс. Возбуждение присутствует. Расстройка (9.76) произвольна, но амплитуда возбуждения $\omega \perp(t)$ подчиняется закону

$$
\omega_{\perp}(t)=\Delta v(t) \tan \Theta,
$$

причем угол $\Theta$ лежит в пределах

$$
0 \leqslant \Theta \leqslant \pi .
$$

В этих условиях гамильтониан прннимает вид

$$
H(t)=v(t) I_{z}+\omega_{2}(t) I_{2}(t),
$$

где

$$
\omega_{2}(t)=\sqrt{\Delta v(t)^{2}+\omega_{\perp}(t)^{2}} .
$$

В данном случае спиновый оператор $I_{y}{ }^{\prime}(t)$ вращается вокруг $I_{z}$ с частотой $v(t)$. Спиновый оператор $I_{2}(t)$ лежит в плоскости $I_{z}, I_{y}{ }^{\prime}(t)$, сохраняя угол $\Theta$ с оператором $I_{z}$. Это и обеспечивается условием (9.79). Движение $I_{m}(t)$ состоит из прецессии вокруг $I_{z}$ и вращения вокруг $I_{2}(t)$ с частотами $v(t)$ и $\omega_{2}(t)$ соответственно.

$$
\Re(t, 0)=\Re_{1}\left(I_{z}, \alpha_{1}(t, 0)\right) \Re_{2}\left(I_{2}(0), \beta_{2}(t, 0)\right),
$$

где в данном случае

$$
\begin{aligned}
& \alpha_{1}(t, 0)=\int_{0}^{t} v\left(t^{\prime}\right) d t^{\prime}, \\
& \beta_{2}(t, 0)=\int_{0}^{t} \omega_{2}\left(t^{\prime}\right) d t^{\prime} .
\end{aligned}
$$

Траектория $I_{2}(t)$ чисто прецессионная. Ей соответствует сигнал

$$
M_{x}(t) \sim-\sin \theta \sin \alpha_{1}(t, 0) .
$$


В случае траекторий, исходящих из начальных состояний $I_{m}(0) \perp$ $\perp I_{2}(0)$, «несущая» (9.84) модулируется движением (9.85). Например, $I_{3}(t)=\sin \Theta \cos \beta_{2}(t, 0) I_{z}+\sin \beta_{2}(t, 0) I_{x}^{\prime}(t)-\cos \Theta \cos \beta_{2}(t, 0) I_{y}^{\prime}(t)$.

Этой траектории соответствует сигнал

$$
\begin{aligned}
M_{x}(t) & \sim 1 / 2(1+\cos \Theta) \sin \left(\alpha_{1}(t, 0)+\beta_{2}(t, 0)\right)- \\
& -1 / 2(1-\cos \Theta) \sin \left(\alpha_{1}(t, 0)-\beta_{2}(t, 0)\right) .
\end{aligned}
$$

В случае произвольного угла между $I_{m}(0)$ и $I_{2}(0)$ сигнал является некоторой суперпозицией сигналов типа (9.86) и (9.88).

Если частоты $\omega_{z}, v, \omega_{\perp}$ постоянны (обыкновенный монорезонанс), то частотный спектр сигнала $M_{x}(t)$ представляет собой триплет. Компонента (9.86) обусловливает центральную частоту $v$, боковые частоты $v \pm \omega_{2}$ происходят от компоненты (9.88).

Амплитуда сигнала с частотой $v$ пропорциональна величине

$$
\sin \Theta=\omega_{\perp} / \sqrt{\Delta v^{2}+\omega_{\perp}^{2}} .
$$

Зависимость этой величины от $\Delta v$ имеет форму типичной спектральной линии с максимумом в точке резонанса и с шириной порядка $\omega_{\perp}$.: Формы боковых линий:

$$
1 / 2\left(1 \pm \Delta v / \sqrt{\left.\Delta v^{2}+\omega_{\perp}^{2}\right)} .\right.
$$

В случае зависящих от времени частот $\omega_{z}(t), v(t), \omega_{\perp}(t)$ основное резонансное поведение все еще сохраняется. Влияние возбуждения заметно только тогда, когда отношение $\Delta v(t) / \omega_{\perp}(t)$ не слишком велико. Однако центр $\omega_{z}(t)$ области резонанса двнжется по шкале частот. В точке резонанса $(9.74) I_{2}(t)=I_{y}^{\prime}(t)$, а $\beta_{2}(t, 0)=2 \alpha_{2}(t, 0)$ описывает нутацию.

Общцй случай. Все частотные характеристики гамильтониана $(9.55)-(9.57) \omega_{z}(t), v(t), \omega_{\perp}(t)$ произвольно зависят от времени. Для определенности рассмотрим только центральную траекторию, предполагая, что гамильтониан приведен к виду (9.65).

Движение по-прежнему состоит из прецессии и нутации. Однако частоты $v_{1}(t), v_{2}(t)$ этих вращений отличаются от частот $v(t)$ и $\omega_{\perp}(t)$ соответственно. Ось нутации $I_{y}{ }^{\prime}(t)$ непрерывно смещается относительно направления $H_{\perp}(t)$.

Тем не менее в условиях (9.60) резонансные свойства возбуждаемости движения сохраняются. Поскольку

$$
\left|v_{2}(t)\right|,\left|v_{3}(t)\right| \leqslant\left|\omega_{\perp}(t)\right|,
$$

то, согласно (9.52), различие частот

$$
\Delta v_{1}(t)=\omega_{z}(t)-v_{1}(t)
$$

имеет тот же порядок величины. Поэтому частота прецессии $v_{1}(t)$ всегда лежит в области резонанса.

Нутация

$$
2 \alpha_{2}(t, 0)=\int_{0}^{t} \omega_{\perp}\left(t^{\prime}\right) \sin \left(\psi\left(t^{\prime}\right)-\alpha_{1}\left(t^{\prime}, 0\right)\right) d t^{\prime}
$$


заметна только тогда, когда частота возбуждения $v(t)$ тоже лежғт в области резонанса. В противном случае интеграл (9.93) исчезает в результате действия быстро осциллирующего множителя.

9.5. 3 а мечание. В области квантовой электроники первые работы о приложении группового подхода принадлежат $\mathrm{Y}$. Х. К о п в ил л е у. В его программном докладе $\left[{ }^{6}\right]$ говорится о связи квантовых динамик с типом их алгебр Ли гамильтонианов, а также (в наших терминах) о представлениях простых динамик в сложных системах. В ряде последующих его работ, сделанных самостоятельно и вместе с сотрудниками, эти идеи прилагаются к разным (существующим и предполагаемым) областям спектроскопии.

\section{Л И Т Е Р А У Р А}

1. Желобенко Д. П. Компактные группы Ли и их представления. М., 1970.

2. B oerner, H. Representations of groups. Amsterdam, 1963.

3. Murnaghan, F. D. The unitary and rotation groups. Washington D. C., 1962.

4. Сини веэ В. Групповой подход в динамике многоспиновых систем. I. - Изв. АН ЭССР, Физ. Матем., 1975 , т. 24 , № 1, с. $35-48$.

5. Ф а й н В. М., Х а н и н Я. И. Қвантовая радиофизика. М., 1965.

6. К опвиллем У. Х. Роль открытия Е. К. Завойским ЭПР в развитии физики. В кн.: Парамагнитный резонанс 1944-1969. (Всесоюз. юбил. конф., Казань, $24-29$ июня 1969 г.). М., 1971 , с. 218.

Институт кибернетики Академии наук Эстонской ССР
Поступила в редакцию $14 / \mathrm{X} 1977$

\section{SINIVEE}

\section{RUHMADE TEOORIA RAKENDAMISEST MITMESPINNISUSTEEMIDE DUNAAMIKA UURIMISEKS. V}

Artiklis on käsitletud kahe nivooga kvantsüsteemi dünaamikat ning jätkatud resonantsi mõiste üldistamist.

\section{SINIVEE}

\section{GROUP APPROACH IN DYNAMICS OF MANY-SPIN SYSTEMS. V}

The dynamics of a two-level quantum system (of a spin $1 / 2$ system) is studied in detail. A further generalization of the resonance concept is given. 